\title{
TRANSMITTING SYSTEMS IN HYDRA
}

AUTHOR(S):

Kass-simon, G.

CITATION:

Kass-simon, G.. TRANSMITTING SYSTEMS IN HYDRA. PUBLICATIONS OF THE SETO MARINE BIOLOGICAL LABORATORY 1973, 20: 583-594

ISSUE DATE:

1973-12-19

URL:

http://hdl.handle.net/2433/175757

RIGHT: 


\title{
TRANSMITTING SYSTEMS IN HYDRA
}

\author{
G. KASS-SIMON ${ }^{1)}$ \\ The University of Wisconsin, Madison, Wisconsin 53706
}

With 9 Text-figures

From the time that it was first discovered by Abraham Trembley in the 18th century, Hydra has had the somewhat dubious privilege of being the most popular of the laboratory coelenterates. Because of this rather special position, it has alternately been raised to the pinnacle of success crowned as a "model animal", only to be plunged into the depths of ill fame and cast aside for being "too specialized." But as always in these matters, the truth is likely to lie somewhere in between. One suspects a sad, but therefore all the more fascinating possibility that Hydra is nothing more nor less than an ordinary, rather common, and perhaps, simply a representative Hydrozoan polyp.

In recent years, ever since George MACKIE so convincingly described epithelial conduction in the Siphonophores (MACKIE, 1965), more and more evidence has been accumulating which indicates that non-nervous conduction plays a very basic and fundamental role in coordinating the behavioral machinery of a great number of organisms (MACKIE, 1971). This certainly is the case for many if not all of the Hydrozoa and also apparently for some Anthozoans (RoBson \& JosEPHSON, 1969 ; MCFARLANE, 1969). And so in its role as typical Hydrozoan polyp, we want to look at the coordinating and transmitting systems of $H y d r a$ and see just what can be attributed to nervous and what to non-nervous electrical activity. In this way, perhaps we can arrive at some sort of cohesive picture of just how these sorts of animals work.

$H y d r a$ spends much of its time simply sitting around in laboratory jars and beakers. Most of this time is spent attached to the bottom or sides of the vessel or sometimes floating at the surface of the water. When it is doing this and when it is left undisturbed, Hydra will remain completely stretched out and elongated for fairly long periods of time. But then, quite suddenly and for no apparent reason, it contracts and with a few pulsations, squeezes itself into a small tight ball. Then it stretches out again.

In the early sixties Passano and McCullough $(1963,1964,1965)$ were able to record electrical correlates for these alternating behaviors. What they found was that as long as the animal was stretched out, small regularly occurring pulses which appeared to come from the basal region of the animal could be recorded. They called these pulses "rhythmic potentials" and postulated their origin from one of several

1) Present address: Department of Zoology, University of Rhode Island, Kingston, R.I. 02881. 
potential pacemakers located in the endodermal nerve net at the base of the Hydra. Sometime later, Gil SHibley at Lawrence University (SHiblex, 1969) showed that an increase in the frequency of rhythmic potential firing was correlated with a tightening of the endodermal lining which caused the animal to twist upward as it elongated. PAssano and MCCullough also found that when the animal shrank, i.e., when the longitudinal muscle processes of the epitheliomuscle cells contracted, a burst of very large pulses emanating from the hypostome could be recorded. They called these "contraction burst pulses" and assigned the ectodermal nerve net as the pathway for their conduction.

But the question of where the pulses were conducted was not really solved and what one wanted to know was not only where they were conducted, but also something of their nature and their origin.

First then, let's look at the rhythmic potential system which produces the regularly occurring pulses accompanying the elongation of the body column.

If one places an electrode inside of a Hydra's mouth, rhythmic potentials appear extremely large and are easy to record; this is not always the case when one attaches suction electrodes to the ectoderm of the animal. Because of their large size, it seemed very likely indeed that rhythmic potential pulses were conducted in the endodermal tissue. The way to prove this was first to show that rhythmic potentials only occur where endodermal tissue is present and fail in places where the endoderm has been removed (KASS-SIMON \& PASSANO, in preparation) and secondly to create a preparation which would only interrupt the suspected rhythmic potential path, but leave other tissue intact (KASS-Simon \& PASSANO, 1969, in preparation). In such preparations, one would expect to find that the rhythmic potentials arising from the pacemaker on one side of the preparation would occur at a different frequency from the rhythmic potentials arising from the pacemaker on the other side of the preparation.

There is a very famous experiment which was performed by Abraham TrembleY in which he turned a Hydra inside out. The next day, Trembley found that the animal had returned to its original orientation. Seeing this, he mistakenly thought that the cells of the endoderm and ectoderm had wandered back to their original position through the mesoglea. Now, it looked as though a good way to make the endoderm accessable so that it could be selectively removed would be to turn a $H y d r a$ inside out in the way TREMBLEY had done. This can easily be accomplished by inserting a pair of forceps into a Hydra's mouth, grasping the basal plate and pulling it back through the mouth; much like taking off a glove. The endoderm is then stripped free down one side of the body column and the Hydra is slit open from foot to mouth along the opposite side. The preparation now consists of two halves of an animal connected only by an ectodermal bridge. Electrodes are placed beneath each tentacle ring or on the tip of a tentacle on each head. Exactly as expected, pulses coming from one head occur at a different rate than pulses coming from the pacemaker of the other head (Fig. 1). Thus it is very clear that when the endoderm is severed, the rhythmic potential 
path is interrupted. Control experiments in which the endoderm was left intact showed no such interruption of rhythmic potential conduction.

The question of what the nature of this endodermal pathway is is still unanswered. And here the evidence pointing to an answer is very much only circumstantial. For the following reasons it seems likely that rhythmic potentials are at least transmitted in the epithelium, if not actually initiated as non-nervous events: 1. MCCONNEL, 1931 and others (LENTZ, 1966) indicate that the nerves in the endoderm are very sparse and that they do not form an actual net. 2. Furthermore, in a series of experiments in which Hydra were subjected to a variety of neuropharmacological inhibitors cholinergic, adrenergic or serotonergic blocking agents did not interfere with the initiation or conduction of rhythmic potentials (KASS-SIMON and PASSANO, in preparation). 3. In preparations where a strip of body tissue provides a path from the pacemaker at the base of the animal to the recording sites at the tentacles, one very frequently, but not
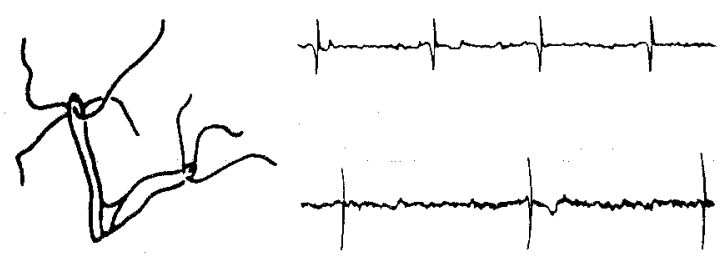

5 SEC.

Fig. 1. Rhythmic potentials from two sides of a bridge-preparation occurring at different frequencies. The pulses fail to be conducted across the endoderm-free bridge.

always, observes that the rhythmic potentials are conducted in such a way that the second, negative going peak arrives precisely at the same time at the two distant channels (Fig. 2). Such synchrony can often but not always be induced by a light shock which seems to have the effect of triggering the instantaneous depolarization of the entire endoderm at rhythmic intervals. In other animals this kind of synchrony is frequently found in electrically coupled nerves but also in the non-nervous electrically active tissue of embryonic and adult vertebrate hearts (DeHAan and GotTLiEB, 1968; WoODBURY and CRILL, 1961). In these tissues, where the myoid cells are electrically coupled, gap junctions and desmosomes connect the individual cells. In Hydra too, gap junctions and desmosomes seem to be quite prevalent. LENTZ at Yale (LeNTZ, 1966) has described septate desmosomes in the apical regions of the endodermal epitheliomuscular cells and SLAUTTERBACK at the University of Wisconsin has recently indicated that gap junctions are to be found between endodermal epithelial cells (personal communication). So, although the evidence is far from conclusive there is a very good chance that the rhythmic potential conducting path is represented by the epitheliomuscular cells of the endoderm.

What of the contraction burst system whose pulses are associated with the longitudinal contraction of the animal? Contraction burst pulses were originally thought 


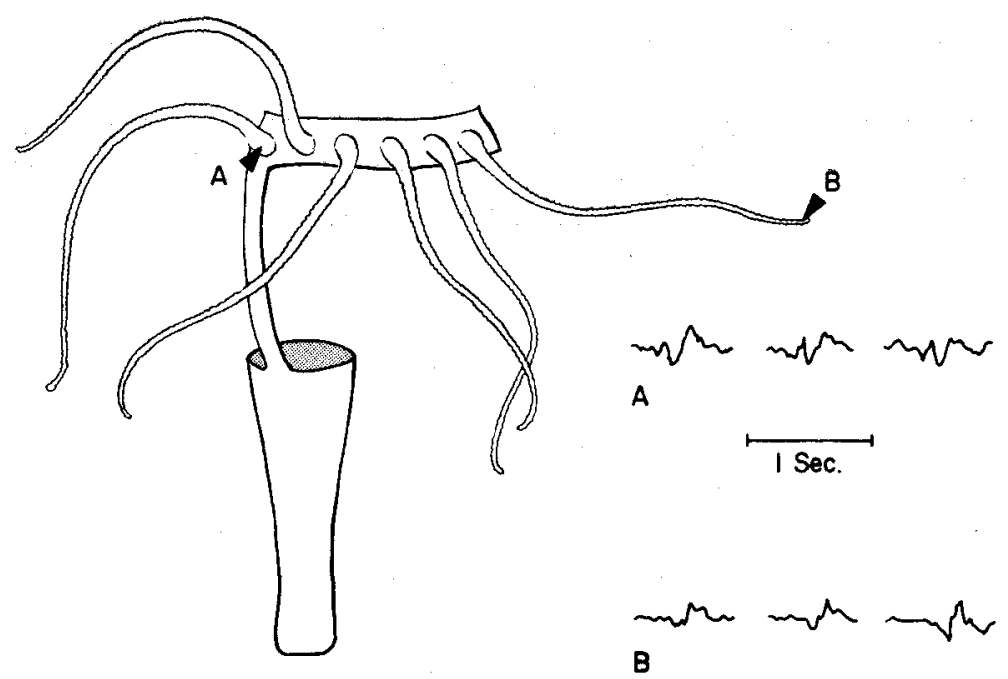

Fig. 2. Consecutive rhythmic potentials recorded from electrodes $(A+B)$ placed at distant tentacles. The slow peaks arrive virtually simultaneously after a light stimulus. Several subsequent pulses were conducted at a finite rate without apparent change of shape.

to arise from the hypostome and be conducted in the ectodermal nerve net (PASSANo and McCullough 1964, 1965).

There is now good reason to believe that contraction burst pulses are also epithelial in nature. For one thing, it has been pointed out by some authors (JOSEPHSON, MACKLIN, 1967 and HorRIDGE, 1968) that these very large pulses (in the order of $30 \mathrm{mv}$ or more) are unlikely to be coming from the fine fibers of the nervous system in such a way as to be able to be recorded with the rather gross suction electrode method.

It also appears that, as in some other polyps (Gonactinia, studied by RoBson, 1971 and Corymorpha, described by PARKER, 1917), the contraction burst conducting system has a distinct longitudinal bias (KASS-SimON, 1970, 1971). It is very easy to observe this bias. If one makes a bridge preparation similar to the one used to demonstrate endodermal rhythmic potential conduction, but this time leaving both ectoderm and endoderm intact, it is possible to observe the following (Fig. 3). If horizontal bridges are narrower than about $100 \mu$ the conduction of the contraction burst pulse and its concomitant behavior frequently fails to cross the bridge. On the other hand, in preparations where bridges of the same widths are vertical, contraction burst pulses and their concomitant behavior are transmitted across the bridge significantly more often. This led to the thought that the conducting path of the contraction burst pulse might be laid out in such a way that pulses travelled down the column in something like a straight-line. Now if this were really the case, it ought to be possible to interfere with the conducting path by cutting the tissue into a maze.

An S-shaped maze can be made by cutting the Hydra open down one side of the 

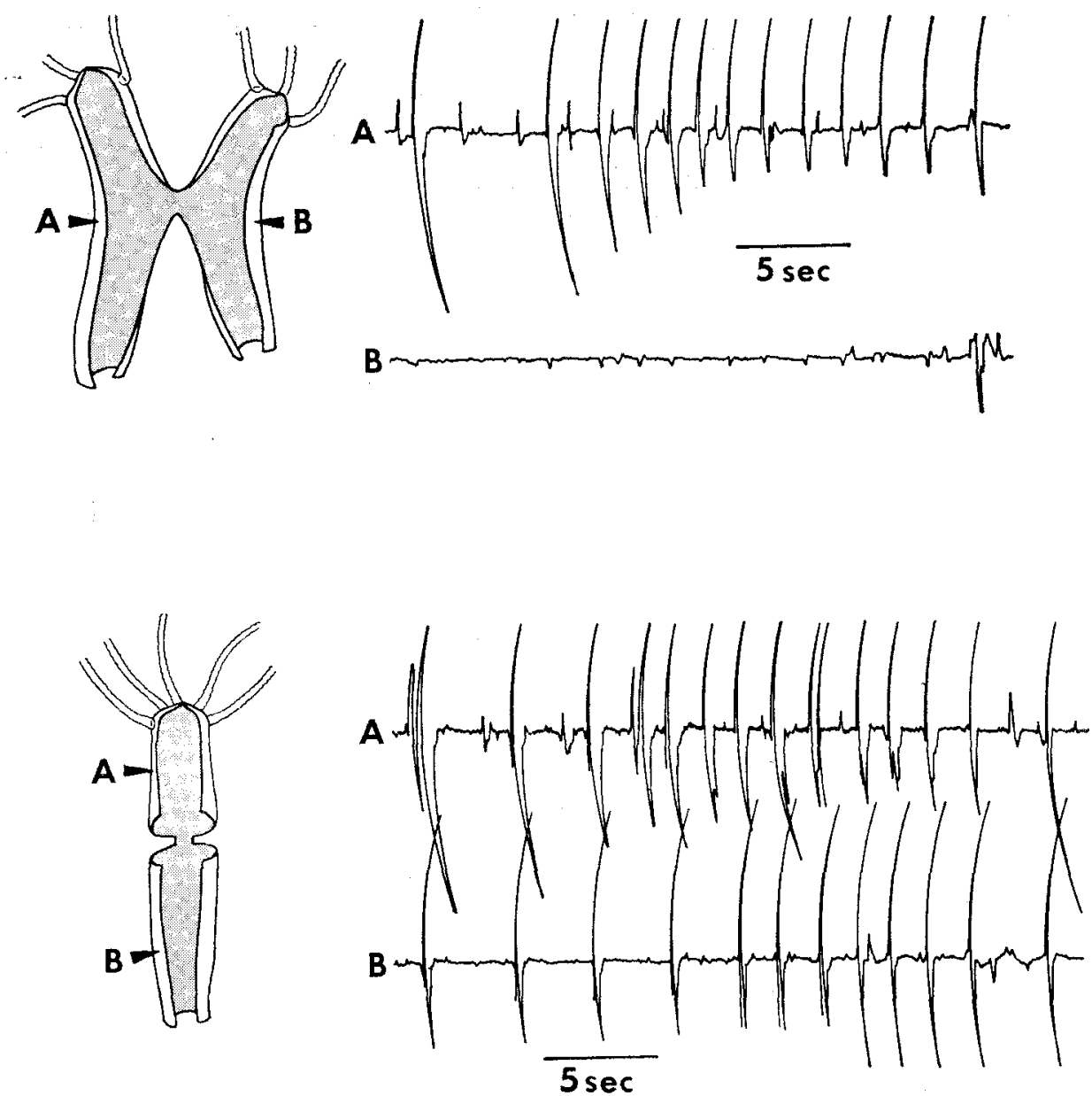

Fig. 3. Conduction of contraction burst pulses in horizontal and vertical bridge preparations. Contraction burst pulses are blocked by a horizontal bridge of $100 \mu$ but are readily conducted across a vertical bridge of the same width. Arrow heads indicate electrode placement. (From Kass-SimON, 1972).

body column and then making horizontal cuts going in opposite directions (Fig. 4). In such preparations pulses originating at the base will get through as far as the middle but will be blocked at the head. Similarly pulses starting at the head will reach the middle but be blocked at the base. In control experiments, if the maze is shaped like the letter E, for bridges of the same width, pulses starting at the base are transmitted to the head significantly more often.

Thus it clearly seems that the conducting path for the contraction burst pulses has a distinct longitudinal bias. And if one considers what that path must look like, on the basis of the experimental results, it seems reasonable to suppose that the hori- 
zontal connectives in the path have a discontinuous, dispersed distribution, while the longitudinal connectives are likely to be continuous, uninterrupted pathways. But the nerve net of Hydra does not appear to fit this description (HADžI, 1909; LENTZ, 1966). The individual elements bear long processes which seem not to have a distinct directionality in the body column. The whole net probably could be turned $90^{\circ}$ in the body of the animal without significantly changing its appearance. The situation, however, is quite different if one looks at the arrangement of the epitheliomuscular cells. These have long processes which run up and down the long axis of the Hydra.

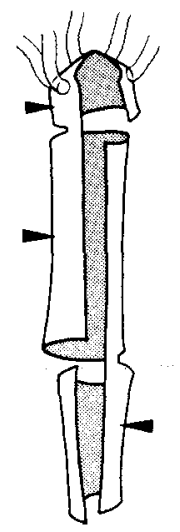

A
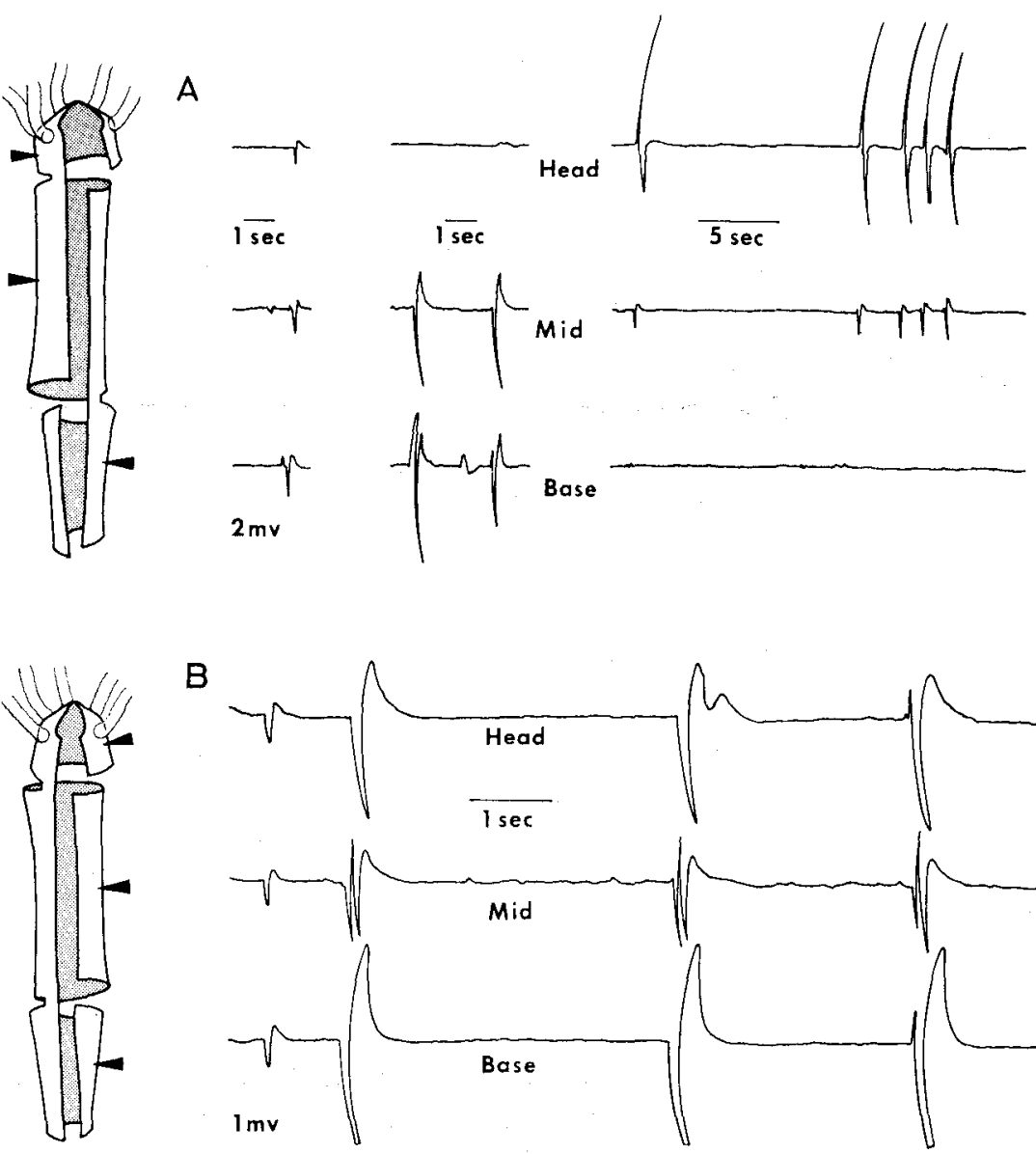

B

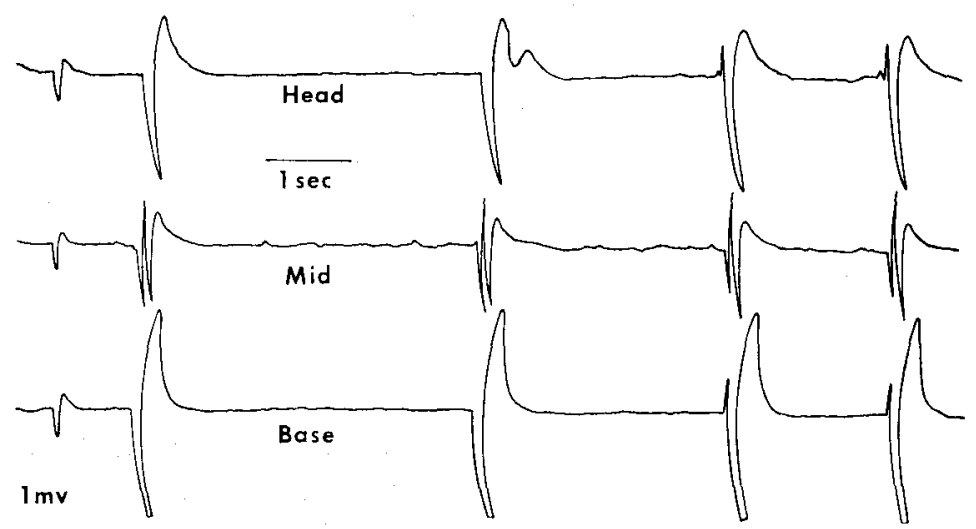

Fig. 4. Conduction of contraction burst pulses in $\mathrm{S}$ and $\mathrm{E}$ preparations. In A contraction burst pulses originating at lower third of the animal is transmitted only as far as the middle section and are blocked by the upper bridge. Later, in the same preparation, contraction burst pulses coming from the upper third are also transmitted only as far as the middle section and are blocked at the lower bridge. In B the contraction burst pulse is transmitted across both bridges. Arrow heads indicate electrode placement. (From Kass-Simon, 1972). 
And it would seem that these processes might be good candidates for the longitudinal path of the contraction burst pulse. This still leaves the questions of the horizontal path and how the pulses are propagated around the body column. A possible anatomical correlate for the proposed discontinuous, dispersed horizontal path exists in the gap junctions which have been found to exist between the myofilaments of adjacent epitheliomuscular cells (WeSRFALl, Yamataka and EnOS, 1970, 1971).

If we look at the conduction velocity of the contraction burst pulse, it appears that as with rhythmic potentials, contraction burst pulses seem to be electrically propagated. Thus if a fairly long conducting path is created by making a threeheaded Hydra it is possible to observe the simultaneous arrival of the contraction burst pulse at each head (Fig. 5). A three-headed preparation is made by slitting a Hydra in half, from mouth to mid-gut, waiting 24 hours and then cutting one of the two resulting heads in half again. (This also is a very old preparation; it was first created by TREMBLEY who managed to get a $H y d r a$ with 7 heads). If one places electrodes on each of the three heads, it is possible to record both contraction burst pulses and tentacle pulses which are associated with tentacle contractions. Tentacle pulses arrive simultaneously at all three electrodes, while contraction pulses often begin the burst sequences with a finite conduction velocity, somewhere in the order of 15 to $30 \mathrm{~cm} / \mathrm{sec}$. And often but not always, ends the burst with several simultaneously
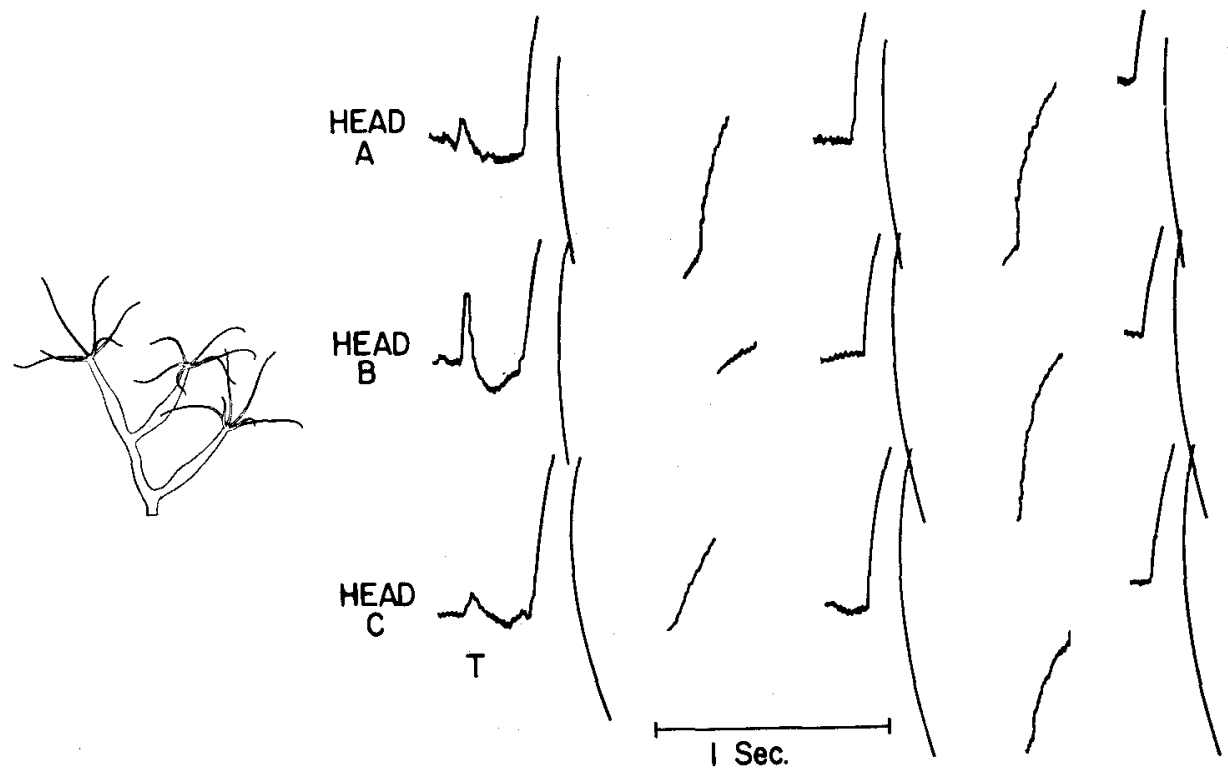

Fig. 5. Contraction burst pulses recorded from electrodes placed just below the tentacle ring of each head. The first pulse of the burst appears to originate from the middle head. Two other pulses toward the end of the burst arrive virtually simultaneously at all three electrodes. $(\mathrm{T}=$ tentacle pulse). 
occurring pulses. What this may imply is that the conducting path between the first and third contraction burst pulses has somehow been altered or facilitated so as to allow the instantaneous depolarization of all the tissue.

It is possible that some unrecorded set of events is being electrically propagated in the nerve net and that these trigger contraction burst pulses simultaneously in each of the three heads; however it seems quite likely, in view of their sometimes rather slow conduction velocity and the probable geometry of their conducting path, that the contraction burst pulse itself is propagated electrically in the myo-epithelium of the ectoderm in a way similar to the epithelial transmission of the rhythmic potentials in the endoderm.

If now, both contraction burst pulses and rhythmic pulses are epithelial as is reasonable to suppose, the question arises as to what the function of the nervous system might be; since, by this time we have practically talked ourselves out of needing a nervous system altogether. The fact is, however, that Hydra does have a nervous system; one where the single units make true synaptic contact with one another as well as with epithelio-muscle cells (Westrall, Yamataka and Enos, 1970, 1971). The question then is, what is this nervous system being used for and what does it do, if it doesn't actually conduct the rhythmic potential and contraction burst pulses?

There has been some indication of late that the nerves in Hydra as elsewhere, e.g. Metridium (RoBSON and JOSEPHSON, 1969) serve as an electrogenic substrate whose activity facilitates and initiates the production of the large pulses we record with suction electrodes.

For one thing, both tentacle pulses and tentacle contractions as well as contraction burst pulses and body contractions are virtually abolished when $H y d r a$ is subjected to cholinergic inhibitors (Kass-Simon and Passano, in preparation). And Robert Singer, 1969 at Case Western Reserve has found that there is an enhancement of motility with drugs which act as anti-cholinesterase agents.

For another, it appears that before the initiation of some kinds of contraction burst, there is a great deal of activity which has the effect of facilitating the initiation of a contraction burst by bringing the responding tissue to threshold (Kass-Simon, 1972). Thus in isolated head preparations, where the body has been cut from the head just below the tentacle ring, activity seems to sweep around the hypostome exciting one tentacle after another until all the tentacles finally contract together. As the activity sweeps around the hypostome, it seems to raise more and more of the hypostomal tissue to firing level, so that each event is larger than the preceding event. Figure 6 shows such facilitation occurring in isolated heads which have been left to heal for about 48 hours. In these preparations the head has elongated and taken on a distinct elliptical shape. The tentacles have spread apart and are at a greater distance from each other than in normal round heads. In these cases it is frequently observed that first one tentacle will fire, then another, then two together, then three, and finally all of the tentacles. And the thought is that some unrecorded series of events is 

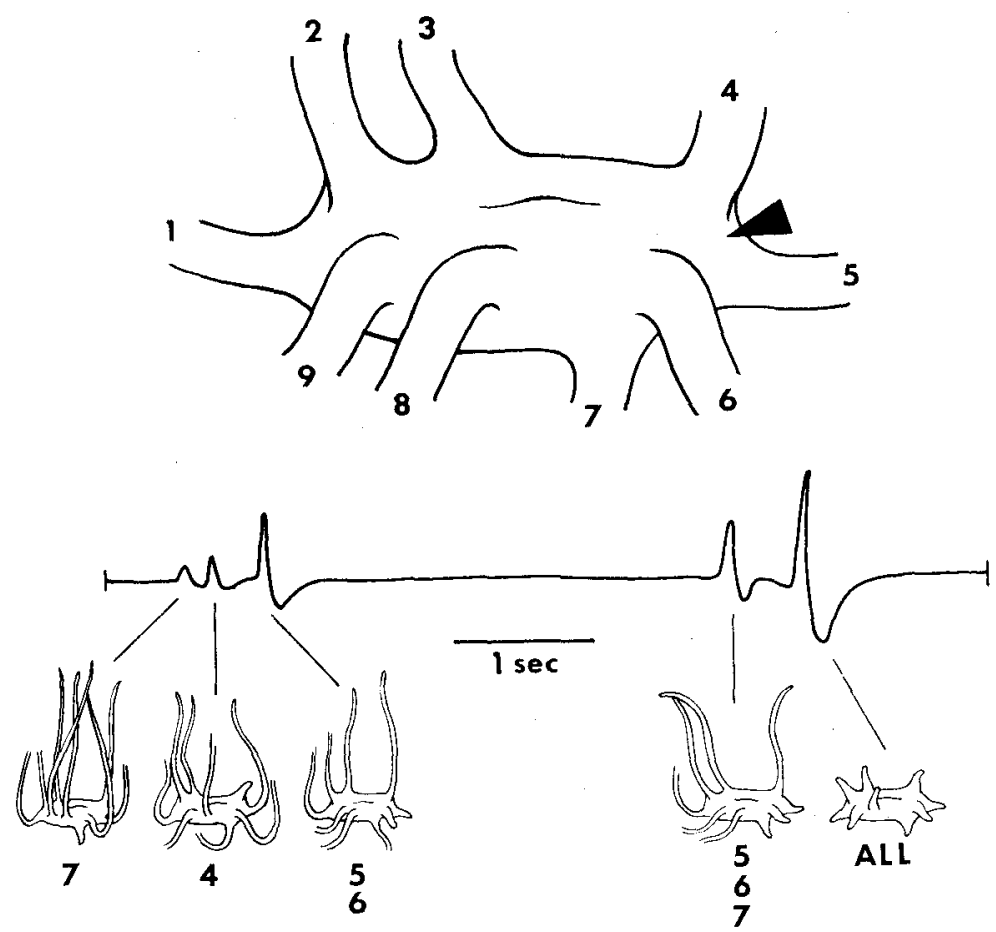

Fig. 6. Crescendo firing in a two-day old isolated head preparation. The recording from only one of the four electrodes (aarrow head) positioned around the head is shown. In this preparation the 'contraction burst' began near tentacles 6, 7, and 8. (From Kass-Simon, 1972).

occurring in the hypostome which triggers each tentacle to fire and which spreads or is conducted in the hypostomal tissue causing ever larger numbers of tentacles to fire. These events may be activity occurring in the nerve net whose neurites have been described to encircle Hydra's hypostome (HADžı, 1909).

The same sort of thing is thought to happen in the initiation of contraction burst pulses and the accompanying body contractions. Activity in the nervous system seems to result in higher and higher levels of excitation until finally a contraction burst pulse is triggered (KASs-Simon and PASSANo, 1969). If a longitudinal strip of body tissue about the width of a tentacle is cut away from the column of the Hydra, together with a single tentacle, and if one attaches an electrode beneath the tentacle, one can observe the following series of events (Fig. 7). First, a small pulse occurs, with no accompanying contraction; then a larger pulse accompanying the contraction of the tentacle. The next pulse is larger still, and the next still larger until finally the entire strip of body tissue contracts. So, what we might say about this and the previous experiment is that contraction bursts occur when sufficient priming or facilitatory activity has brought the responding epithelium to threshold. 


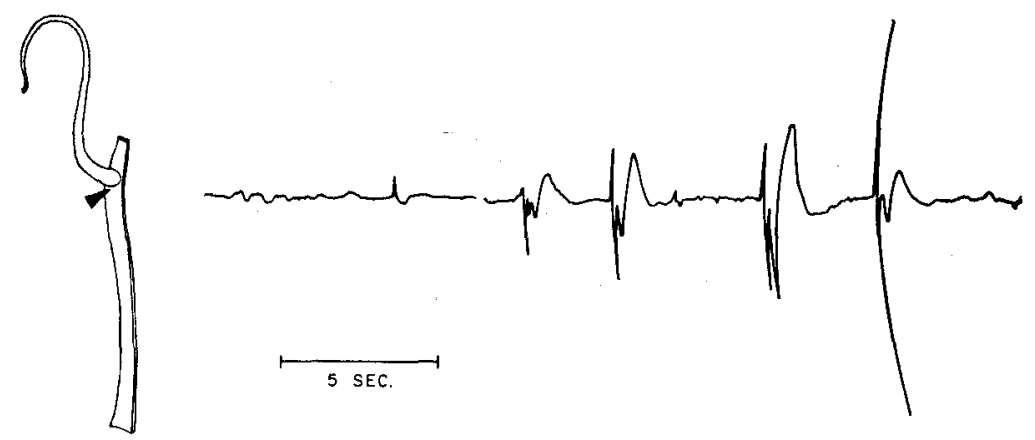

Fig. 7. Facilitation in a body strip-tentacle preparation. Increasingly larger pulses accompany the contraction of a single tentacle and culminate in the contraction of the strip of body wall.

We can see how this sort of facilitatory activity coordinates tentacle and body contractions in intact animals. The spread of excitation and the initiation of both tentacle and body contractions can be seen in the following preparation (Fig. 8). An animal is cut lengthwise in half, from basal plate to just below the tentacles. Within 24 hours each half forms a complete body column. Then, just before the experiment, one side of the hypostome is cut open to form a flat sheet of tissue. Electrodes are then placed on each body column near the base and two other electrodes are placed between tentacles on each side of the preparation. In these preparations, the following sequence of events can be observed. First, there will be some tentacle activity on one side of the head. Then the body underneath the active tentacles contracts. Then the excitation spreads to the other side of the hypostome causing the tentacles there to
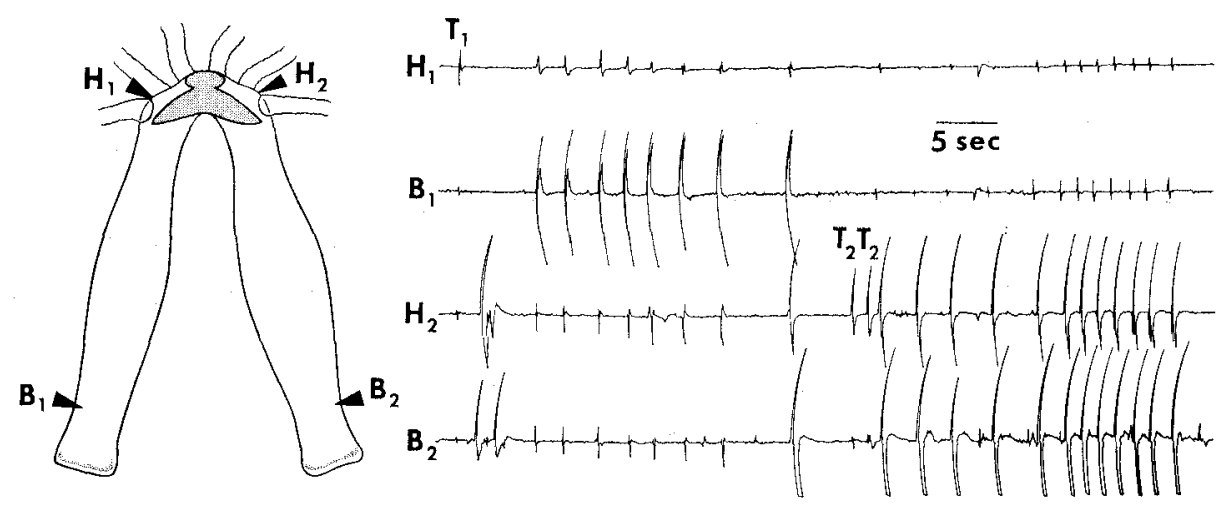

Fig. 8. Contraction burst pulse initiation and conduction in split body preparations. The sequence begins with a tentacle contraction on one side of the preparation $\left(T_{1}\right)$ followed by a body contraction under that tentacle. The second body column begins to contract before the first column has ended its contraction burst. Arrow heads indicate electrode placement. (From Kass-Simon, 1972). 
contract, followed by the contraction of the body on that side. If the tentacles in the middle contract it is often the case that both body columns will contract together.

We can now summarize what may happen in the initiation and propagation of contraction burst pulses. It is quite likely that what is happening goes something like this: Spontaneous activity in the hypostomal nerve net at any neuromuscular site close to or at the base of a tentacle triggers a pulse in the epithelium of the tentacle causing it to contract. Each tentacle event facilitates the spread of subsequent events either via the hypostomial epithelium or the nerve net. When a site on the conducting epithelium has reached threshold, it produces a contraction burst pulse which is propagated down the muscle processes of the epitheliomuscle cells and horizontally over the ectoderm via the scattered unevenly distributed gap junctions between the ectodermal epitheliomuscle cells.

The histological picture presented by Wesrfall, Yamataka and EnOS, 1971 seems to fit the requirements of this model very well. Nerves have been described to synapse with other nerves and with epitheliomuscle cells; and gap junctions are found between the myofilament-bearing regions of adjacent cells.

So although it is not at all clear that Hydra is a representative Hydrozoan, its coordinating systems seem to have many of the attributes which are just now being described for a number of other coelenterates: Non-nervous transmission of electrical events appears to play as important a role in controlling $H y d r a$ 's behavioral repertoire as does the activity occurring in its nervous system. And this seems also to be the case in many other polyps and medusae.

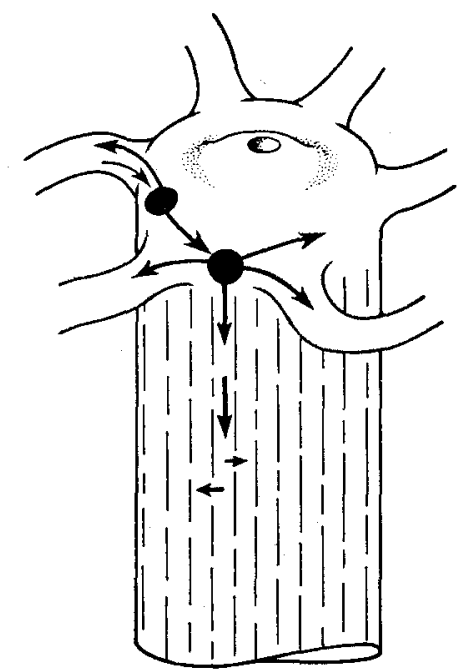

Fig. 9. A conceptualization of the pulse initiation sites and conduction pathways in Hydra. Closed circles represent initiation sites. Arrows indicate transmission of electrical activity. The conducting system in the column is represented as a series of continuous longitudinal paths with discontinuous, randomly spaced horizontal transmission sites. (From KASs-SimON, 1972). 


\section{Acknowledgements}

The work reported here was performed in the laboratory of L. M. PASSANo and supported by NSF Grant No. B012421. Thanks are due Cheryl Hughes for preparing the text figures, Donald Chander for photographing them and Linda MCConnell for typing the manuscript.

\section{REFERENCES}

DeHaAn, R. L. and GotTrieb, S. H. 1968. The electrical activity of embryonic chick heart cells isolated in tissue culture singly or in interconnected cell sheets. J. Gen. Physiol. 52: 643-663.

HADŽI, J. 1909. Über das Nervensystem von Hydra. Arb. Zool. Inst. Univ. Wien. 17: 225-269.

Horridge, G. A. 1968. Interneurons. W. H. Freeman \& Co. London and San Francisco.

Josephson, R. K. and MACKLIN, M. 1967. Transepithelial potentials in Hydra. Science 156: 16291631.

Kass-Simon, G. 1970. Multiple excitation sites and straight-line conduction in the contraction burst system of Hydra. Amer. Zool. 10: 505.

KAss-Simon, G. 1972. Longitudinal conduction of contraction burst pulses from hypostomal excitation loci in Hydra attenuata. J. Comp. Physiol. (Z. Vergl. Physiol.) 80: 29-49.

Kass-Simon, G. and Passano, L. M. 1969. Conduction pathways in Hydra. Amer. Zool. 9: 113.

LeNTz, T. L. 1966. The Cell Biology of Hydra. John Wiley \& Sons, Inc. N. Y.

MACKIE, G. O. 1965. Conduction in the nerve-free epithelia of siphonophores. Amer. Zool. 5: 439453.

Mackie, G. O. 1970. Neuroid conduction and the evolution of conducting tissues. Quart. Rev. Biol. 45: 319-332.

MCConnell, C. H. 1931. A detailed study of the endoderm of Hydra. J. Morphol. 53: 249-263.

MCFARLANe, I. D. 1969. Two slow conduction systems in the sea anemone Calliactis parasitica. J. exp. Biol. 51: 377-386.

PARKer, G. H. 1917. The activities of Corymorpha. J. Exp. Zool. 24: 303-331.

Passano, L. M. and McCullough, C. B. 1963. Pacemaker hierarchies controlling the behavior of hydras. Nature (Lond.): 1174-1175.

Passano, L. M. and McCullough, C. B. 1964. Co-ordinating systems and behavior in hydra. I. Pacemaker system of the periodic contractions. J. exp. Biol.: $41: 643-664$.

Passano, L. M. and McCullough, C. B. 1965. Co-ordinating systems and behavior in hydra. II. The rhythmic potential system. J. exp. Biol. 42: 205-231.

RoBson, E. A. 1971. The behavior and neuromuscular system of Gonactinia prolifera, a swimming sea-anemone. J. exp. Biol. 55: 611-640.

Robson, E. A. and Josephson, R. K. 1969. Neuromuscular properties of mesenteries from the sea-anemone Metridium. J. exp. Biol. 50: 151-168.

SHIBLEY, G. 1969. Gastrodermal contractions correlated with rhythmic potentials and prelocomotor bursts in Hydra. Amer. Zool. 9: 586.

SINGER, R. H. 1969 . The effect of neuropharmacological drugs on the light response of Hydra piradi.

Woodbury, J. W. and Crill. 1961. On the problem of impulse conduction in the atrium. In Nervous Inhibitors. E. Florey, ed. Pergamon Press, New York. 